\title{
Modic Changes and Disc Degeneration Caused by Inoculation of Propionibacterium acnes inside Intervertebral Discs of Rabbits: A Pilot Study
}

\author{
Zhe Chen, ${ }^{1,2}$ Yuehuan Zheng, ${ }^{3}$ Ye Yuan, ${ }^{1}$ Yucheng Jiao, ${ }^{1,2}$ Jiaqi Xiao, \\ Zezhu Zhou, ${ }^{5}$ and Peng Cao ${ }^{1,2}$ \\ ${ }^{1}$ Department of Orthopedics, Ruijin Hospital, Shanghai Jiao Tong University School of Medicine, Shanghai 200025, China \\ ${ }^{2}$ Shanghai Key Laboratory for Prevention and Treatment of Bone and Joint Diseases with Integrated Chinese-Western Medicine, \\ Shanghai Institute of Traumatology and Orthopedics, Ruijin Hospital, Shanghai Jiao Tong University School of Medicine, \\ Shanghai 200025, China \\ ${ }^{3}$ Department of Orthopedics, Ruijin Hospital North, Shanghai Jiao Tong University School of Medicine, Shanghai 201821, China \\ ${ }^{4}$ Department of Medical Microbiology and Parasitology, Shanghai Jiao Tong University School of Medicine, Shanghai 200025, China \\ ${ }^{5}$ Department of Orthopedic Surgery, Xinhua Hospital, Shanghai Jiao Tong University School of Medicine, Shanghai 200092, China
}

Correspondence should be addressed to Zezhu Zhou; drzhouzezhu@163.com and Peng Cao; dr_caopeng8@163.com

Received 13 December 2015; Accepted 14 January 2016

Academic Editor: Panagiotis Korovessis

Copyright (C) 2016 Zhe Chen et al. This is an open access article distributed under the Creative Commons Attribution License, which permits unrestricted use, distribution, and reproduction in any medium, provided the original work is properly cited.

\begin{abstract}
Purpose. To investigate whether $P$. acnes could induce disc degeneration and Modic changes when inoculated into the discs of rabbits. Method. A wild-type strain of P. acnes isolated from a patient associated with Modic change and disc degeneration was inoculated into the intervertebral discs of rabbits. Meanwhile, $S$. aureus was injected into the discs to establish a model of discitis as the comparison and a standard strain of $P$. acnes was inoculated as the control. MRI and histological change were observed. Results. Both the P. acnes-inoculated and $S$. aureus-inoculated rabbits showed hyperintense signals at endplates and hypointense signals at nucleus pulposus on T2WI. However, P. acnes only resulted in moderate disc degeneration and endplates rupture in histological examination, which was different from the pathological change of discitis caused by S. aureus. In addition, higher death rates $(2 / 3$ versus 0/5) were observed in S. aureus-inoculated rabbits. Conclusion. Compared to S. aureus, the pathological change caused by $P$. acnes would be considered as Modic-I change and disc degeneration rather than a discitis.
\end{abstract}

\section{Introduction}

Modic changes are the successive event of disc degeneration [1], presenting as signal intensity changes at cartilage endplates and subchondral bone in MRI [2,3]. Many studies have claimed a strong connection between Modic changes and nonspecific low back pain and that is why so much attention was given to this pathological change $[4,5]$. Unfortunately, the exact pathogenic mechanism of Modic changes is still unclear until now.

One of the explanations was the bacterial mechanism. It was reported that some low-virulent anaerobic bacteria could be isolated from the intervertebral discs only with degeneration but without pyogenic change $[6,7]$. Subsequently, the low-virulent anaerobic bacteria were suggested as the pathogens for Modic changes and low back pain [8,9]. A randomized controlled trial showing that patients acquired excellent pain relief and Modic-I changes attenuation after oral antibiotics treatment further validated this theory [10].

Among those low-virulent anaerobic bacteria, Propionibacterium acnes ( $P$. acnes) was the major and most frequent isolated bacterium, accounting for approximately $85 \%$, so that it ought to be the most possible pathogen for Modic change and low back pain $[6,8]$. It is an anaerobic-aerotolerant Gram-positive rod-shaped bacterium, residing in the skin, oral cavity, and conjunctiva as part of the normal flora [11]. More importantly, it was also an opportunistic pathogen responsible for series of bone and joint infection [11]. 
TABLE 1: Animal group information.

\begin{tabular}{|c|c|c|c|c|c|}
\hline & $\begin{array}{l}\text { Inoculated } \\
\text { bacterial type }\end{array}$ & Numbers & $\begin{array}{l}\text { Inoculated } \\
\text { segment }\end{array}$ & Bacterial load & Dead or not \\
\hline Group A & $\begin{array}{l}\text { The wild-type } \\
\text { strain of } P \text {. acnes } \\
\text { isolated from } \\
\text { patient }\end{array}$ & 3 & $\begin{array}{l}\text { L6 L7 } \\
\text { L5 L6 }\end{array}$ & $\begin{array}{l}\text { P. acnes suspension in sterilized } \\
\text { TSB without bovine serum at } 1 \times \\
10^{7} \mathrm{CFU} / \mathrm{mL} \text { with } 25 \mu \mathrm{L} \\
\text { Sterilized TSB without bovine } \\
\text { serum with } 25 \mu \mathrm{L}\end{array}$ & All alive until the end of follow-up \\
\hline Group B & $\begin{array}{l}\text { The standard strain } \\
\text { of } P \text {. acnes (ATCC } \\
6919 \text { ) }\end{array}$ & 2 & $\begin{array}{l}\text { L6 L7 } \\
\text { L5 L6 }\end{array}$ & $\begin{array}{l}\text { P. acnes suspension in sterilized } \\
\text { TSB without bovine serum at } 1 \times \\
10^{7} \mathrm{CFU} / \mathrm{mL} \text { with } 25 \mu \mathrm{L} \\
\text { Sterilized TSB without bovine } \\
\text { serum with } 25 \mu \mathrm{L}\end{array}$ & All alive until the end of follow-up \\
\hline Group C & $\begin{array}{l}\text { The standard strain } \\
\text { of } S \text {. aureus (ATCC } \\
\text { 25923) }\end{array}$ & 3 & $\begin{array}{l}\text { L6 L7 } \\
\text { L5 L6 }\end{array}$ & $\begin{array}{l}\text { S. aureus suspension in sterilized } \\
\text { saline at } 1 \times 10^{7} \mathrm{CFU} / \mathrm{mL} \text { with } 25 \mu \mathrm{L} \\
\text { Sterilized saline with } 25 \mu \mathrm{L}\end{array}$ & $\begin{array}{l}\text { One died at the fourth week } \\
\text { One died at the fifth week } \\
\text { One alive until the end of follow-up }\end{array}$ \\
\hline
\end{tabular}

Nevertheless, there has been controversies and argumentation against the relationship between bacteria and Modic changes, highlighting that the isolated $P$. acnes was the contamination during tissue harvest rather than the original colonization inside intervertebral discs [12]. In addition, the lack of deterministic causal evidence based on animal studies was another major defect [13]. To solve this problem, Koch's Postulates, which demonstrate that pathogenic microbes isolated from patients could cause the same disease in animal models, were a reasonable method $[14,15]$.

Therefore, the primary goal of this investigation was to test whether the wild-type strain of $P$. acnes isolated from the intervertebral disc of patient associated with Modic changes and disc degeneration has the ability to induce the same pathological change when inoculated into the discs of rabbits. In the meantime, a pyogenic discitis model caused by Staphylococcus aureus (S. aureus) was established to make a comparison and a standard strain of $P$. acnes was used as the control. To our knowledge, this is the first animal experiment to demonstrate the possible causative relationship between $P$. acnes and Modic changes, which is very important to reveal the exact role low-virulence anaerobic bacteria are playing in intervertebral discs.

\section{Materials and Methods}

2.1. Animals. The harvest of intervertebral disc from human being was approved by the clinical Ethics Committee of Ruijin Hospital, Shanghai Jiao Tong University School of Medicine. Animal experiments were approved by the Animal Ethics Committee of Shanghai Jiao Tong University School of Medicine. A total of eight New Zealand rabbits weighing around $2.0 \sim 2.5 \mathrm{~kg}$ each were included in this study (Table 1). They were fed with regular water and food and kept for at least one week before the surgery for acclimatization.

2.2. Isolation and Identification of the Wild-Type Strain of P. acnes. A wild-type strain of $P$. acnes was isolated from a patient who initially took part in the epidemiological study about the relationship between $P$. acnes and disc degeneration [16]. The patient, a 48-year-old Chinese male, underwent discectomy due to sciatica and low back pain and did not show any symptoms of discitis (no fever, no body weight loss, and no abnormal ESR). MRI revealed a severe disc herniation and Modic change at intervertebral discs of L4 5 (Figures 1(a) and $1(\mathrm{~b}))$.

For the process of isolation of the bacterium, disc material of L4 L5 was harvested under stringent sterilized situation and some muscle and ligaments around the disc were also obtained as contamination marker. All samples (disc material and control samples) were enriched in a $9 \mathrm{~mL}$ sterilized Tryptone Soya Broth (TSB, BD, NJ, USA) mixed with $1 \mathrm{~mL}$ of bovine serum (Gibco, Life Technologies, CA, USA). The broth was cultured inside an anaerobic Glove Box device (Yuejin Medical Instruments Corporation, Shanghai, China) for fourteen days with circumstance of $37^{\circ} \mathrm{C}, 80 \% \mathrm{~N}_{2}-10 \%$ $\mathrm{CO}_{2}-10 \% \mathrm{H}_{2}$ mixed gas. When limpid broth turned into cloudy, it was transferred into the Anaerobic Blood Agar (Beiruite Bio-technology, Zhengzhou, China) for another three-day culture under the same anaerobic circumstance.

Following previous method [8], the purified single bacterial colony was identified using Gram's staining, analytical profile index (API) biochemical analysis of Rapid ID 32A (bioMérieux, France), and polymerase chain reaction (PCR) amplification of $16 \mathrm{SrDNA}$. The procedures of $16 \mathrm{SrDNA}-\mathrm{PCR}$ followed previous method $[8,16]$. Finally, a wild-type strain of $P$. acnes was isolated from the intervertebral disc of the patient and no bacterium was found at the muscle and ligament, suggesting that the isolated $P$. acnes originated from the intervertebral discs rather than from the contamination of the incision. Moreover, this microbe was confirmed as $P$. acnes by these three identification methods (Figure 1(c)).

2.3. Preparation of Bacterial Inoculum. The wild-type strain of $P$. acnes harvested from a single colony on the agar was washed for three times using TSB without bovine serum and then suspended in the same solvent (sterilized TSB without bovine serum) with adjusting the concentration to $1 \times 10^{7} \mathrm{CFU}$ (Colony-Forming Units) per $\mathrm{mL}$ using the plate count method. In the meantime, a standard strain of $P$. acnes was obtained from the Guangdong Microbiology Culture Center (ATCC: 6919, GIM: 1.243, Guangdong, China) and 


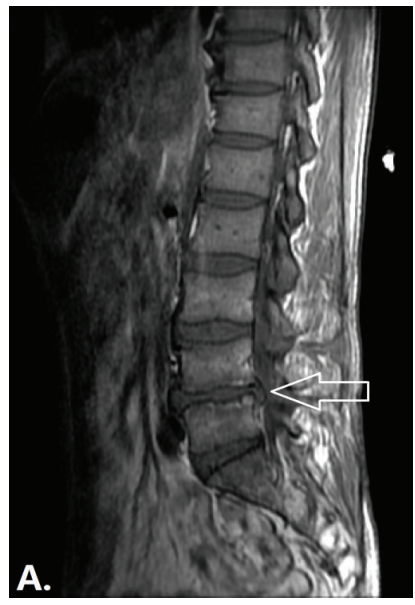

(a)

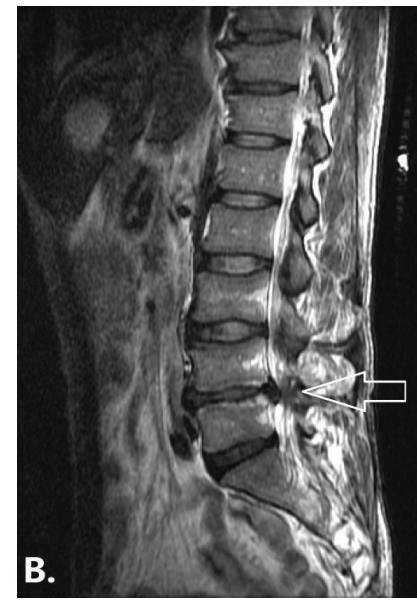

(b)

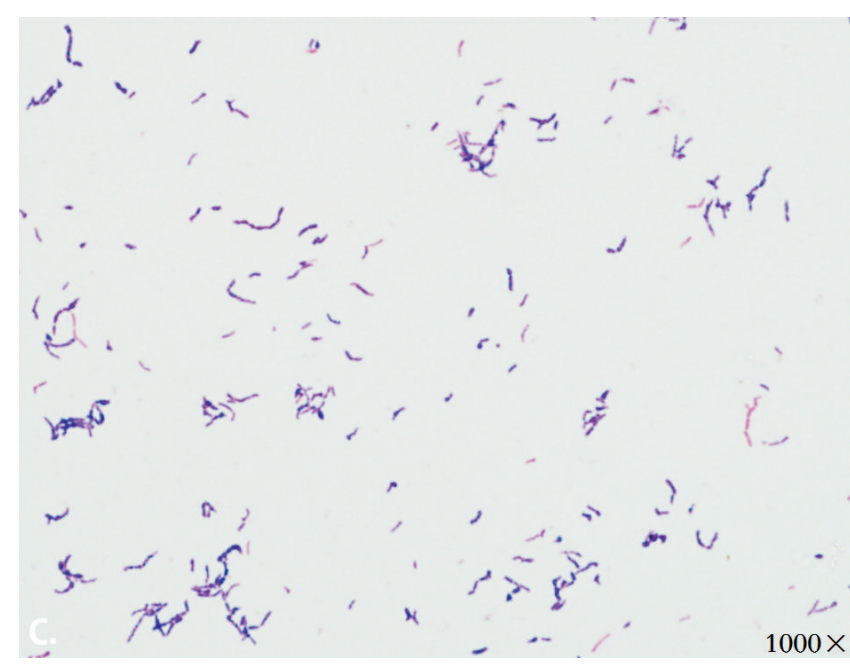

(c)

FIGURE 1: The wild-type strain of $P$. acnes was isolated from the patient associated with severe disc degeneration and Modic change at the segment of L4 L5. (a) (b) MRI examination of the patient showed signal intensity changes on the segment of L4 L5 at the cartilage endplate on T1WI and T2WI (a, b), suggesting a Modic change (b). Both were indicated by a white arrow. Meanwhile, severe disc degeneration and disc herniation were found at the same segment. (c) Gram's staining depicted a Gram-positive rod-shaped bacterium after three-day culture in anaerobic blood plate.

prepared into the same suspension as the method described above. Additionally, a single colony of $S$. aureus (ATCC 25923, granted by Department of Medical Microbiology and Parasitology, Shanghai Jiao Tong University School of Medicine) harvested from Columbia blood agar plate was suspended in sterilized saline with the same concentration of $1 \times 10^{7} \mathrm{CFU}$ per $\mathrm{mL}$.

2.4. Surgical Procedure. All rabbits were anesthetized using $2.5 \%$ sodium pentobarbital with $1 \mathrm{~mL} / \mathrm{kg}$ via auricular vein and were then placed in right lateral position. The skin was shaved and sterilized carefully with povidone iodine for three times. Prior to the surgery, the desired vertebrae (L5, L6, and L7) and intervertebral discs (L5 L6 and L6 L7) were identified using CT. Meanwhile, the diameters of the desired intervertebral discs on coronal plane were measured using CT to determine the depth of penetration.

A posterolateral retroperitoneal approach was conducted to expose the desired vertebrae (L5, L6, and L7) and discs (L5 L6 and L6 L7) [17]. Before inoculation, plenty of sterilized saline was used to wash the incision to prevent contamination. A microsyringe with 28-gauge needle (Hamilton, Nevada, USA) was used for inoculation and the penetration depth was fixed at $5 \sim 6 \mathrm{~mm}$ based on the measured parameters. A volume of $25 \mu \mathrm{L}$ wild-type strain or standard strain of $P$. acnes suspension was inoculated into the nucleus pulposus at the segment of L6 L7 and $25 \mu \mathrm{L}$ of sterilized TSB without bovine serum was injected at the segment of L5 L6 as internal control at the same time (Table 1). The holes of penetration were sealed with bone wax to prevent the leakage of inocula and the fascia and skin were closed layer by layer with silk sutures. Collectively, three animals received the wild-type strain of P. acnes isolated from the patient and the other two animals were inoculated with the standard strain of $P$. acnes. With the same method, the other three rabbits received the inoculation of $25 \mu \mathrm{L} \mathrm{S}$. aureus suspension at the segment of L6 L7 (Table 1). All animals were fed regularly and no antibiotics were used before and after the surgery.

2.5. MRI Examination. MRI was performed before and every two weeks after the inoculation until the end of follow-up at the eighth week. Briefly, animals were fasted closely on a wooden plate at prone position after deep anaesthetization and scanned with knee coil in a 3.0-T GE HDxt Signa/MRI system (GE Corporation, Connecticut, USA). The parameters were as follows: T1W TE/TR, $8.4 \mathrm{~ms} / 540 \mathrm{~ms}$, T2W TE/TR, $48.2 \mathrm{~ms} / 2000 \mathrm{~ms}$, slice thickness, $3 \mathrm{~mm}$, and Field of View, $20 \times 20$. All MRI images were evaluated by two authors independently (Z. Chen and Z. Zhou). According to Pfirrmann classification system, the degree of intervertebral discs degeneration was evaluated and classed into five grades [18].

2.6. Histological Examination. Animals were euthanized at the eighth week for histological examination. Briefly, intervertebral discs with endplates were fixed in $4 \%$ formaldehyde for 24 hours and then decalcified with EDTA (Ethylenediaminetetraacetic Acid) for three months, processed with routine paraffin embedding, and sectioned at $5 \mu \mathrm{m}$. Hematoxylin and eosin staining was conducted and digital pictures were captured under the magnification of $100 x$.

\section{Results}

3.1. Signal Changes in MRI at P. acnes-Inoculated and S. aureus-Inoculated Intervertebral Discs. No abnormal signals were found before the surgery (Figures 2(a) and 2(b)). 


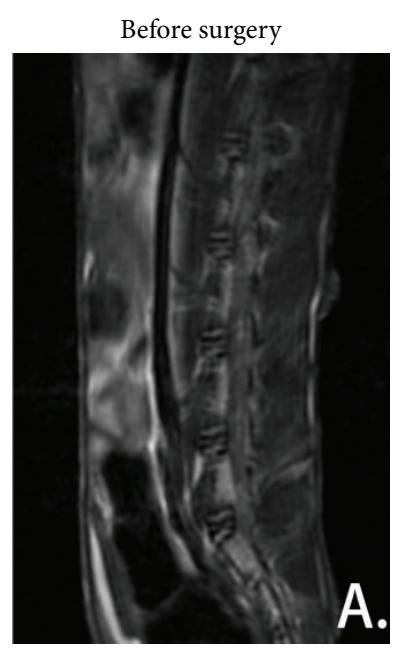

(a)

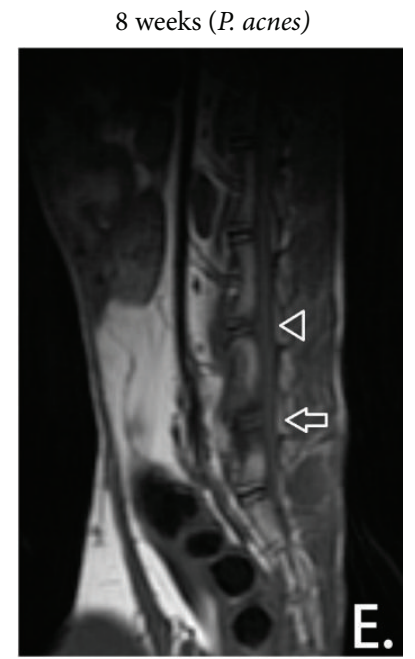

(e)

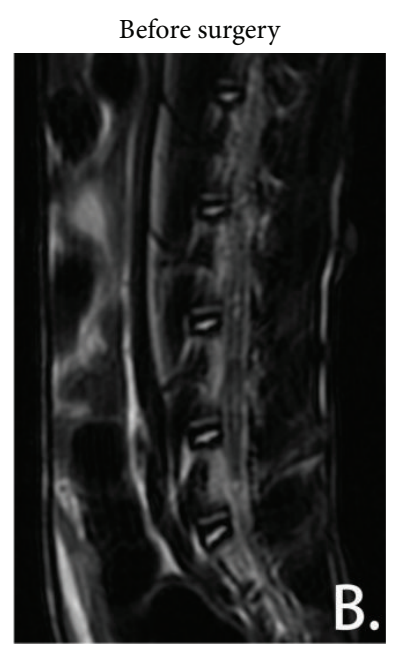

(b)

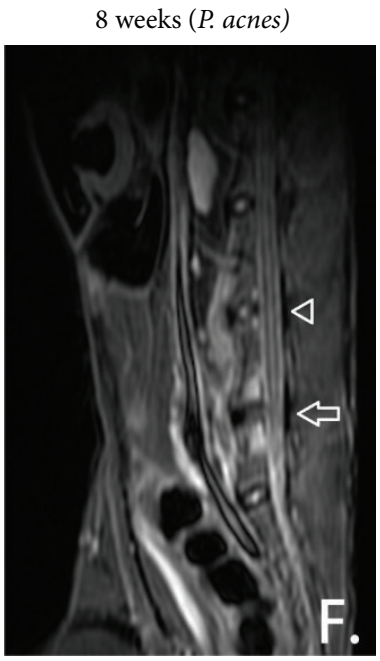

(f)

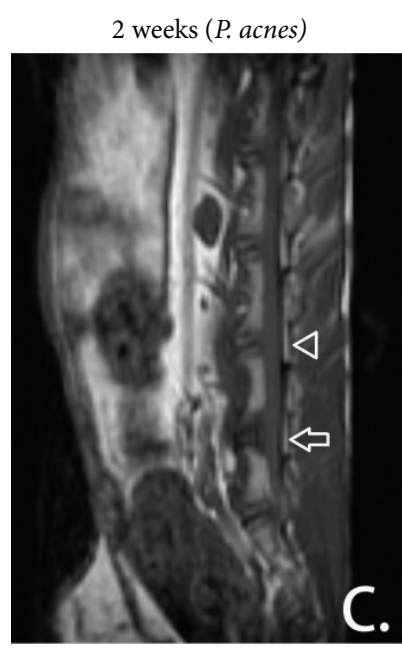

(c)

8 weeks (S. aureus)

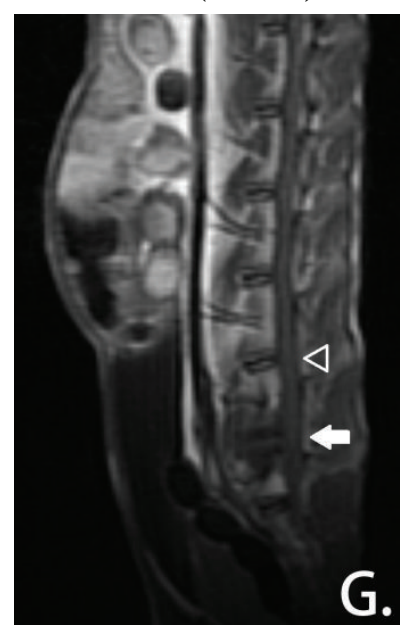

(g)

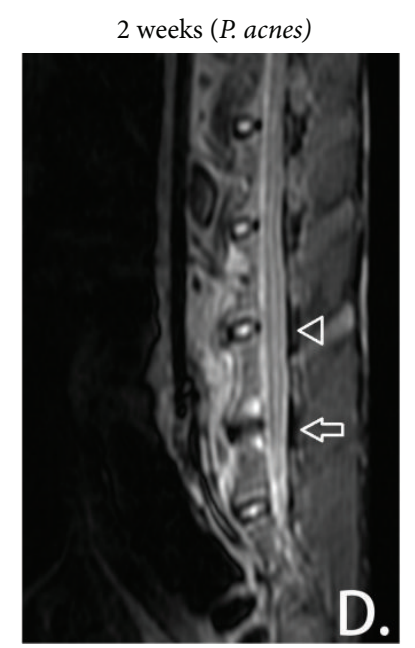

(d)

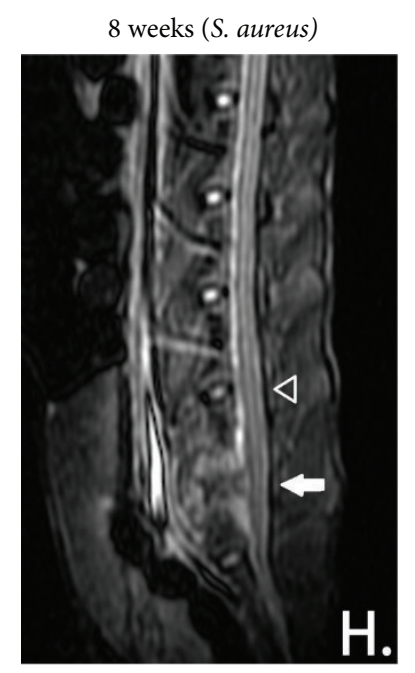

(h)

Figure 2: Signal changes were observed after the inoculation of the isolated wild-type strain of P. acnes and S. aureus. (a) (b) Before the surgery, there was not any abnormality at T1WI and T2WI. (c) (d) Since the second week, an obvious hypointense signal was observed on T1WI (c) and the hyperintense signal was found on T2WI (d) at the P. acnes-inoculated segment (L6 L7, indicated by a white arrow). Meantime, hyperintense signals of nucleus pulposus in T2WI disappeared, suggesting disc degeneration. (e) (f) The signal changes remained constant until the eighth week at the end of follow-up. The volume of signal changes had increased from the second week to the eighth week. (g) (h) The hyperintense signal changes were also observed in the S. aureus-inoculated segment (L6 L7, indicated by a white filled arrow) on T1WI (g) and T2WI (h); however, the definition of the endplates and vertebral body was less intact and more severe inflammatory signal changes were found. No significant signal changes were observed at the internal control segment of L5 L6 in all groups (indicated by a white triangle).

However, signal changes of cartilage endplates were detected at both the wild-type strain and standard strain of $P$. acnes-inoculated segment (L6 L7) since the second week, revealing hypointense signals at T1WI and hyperintense signals at T2WI (Figures 2(c) and 2(d)). Furthermore, the normal hyperintense signal of nucleus pulposus shifted into hypointense signal on T2WI, demonstrating a "black disc" which was considered as an indication of disc degeneration. All of these abnormal signals remained constant for eighth week until the euthanasia of the animals and the volume of signal changes had increased from the second week to the eighth week (Figures 2(e) and 2(f)). By contrast, the TSB-inoculated internal control segment was normal during the observation (Figures 2(c)-2(f)). According to Pfirrmann classification system, the severity of the intervertebral disc degeneration was V or IV grades in all of the wild-type strain or standard strain of $P$. acnes-inoculated animals.

In addition, similar signal changes were also found in the $S$. aureus-inoculated intervertebral discs, presenting as hyperintense signals at the endplates and hypointense signals at the nucleus pulposus on T2WI. However, the definition of the endplates and vertebral body was less intact and more 


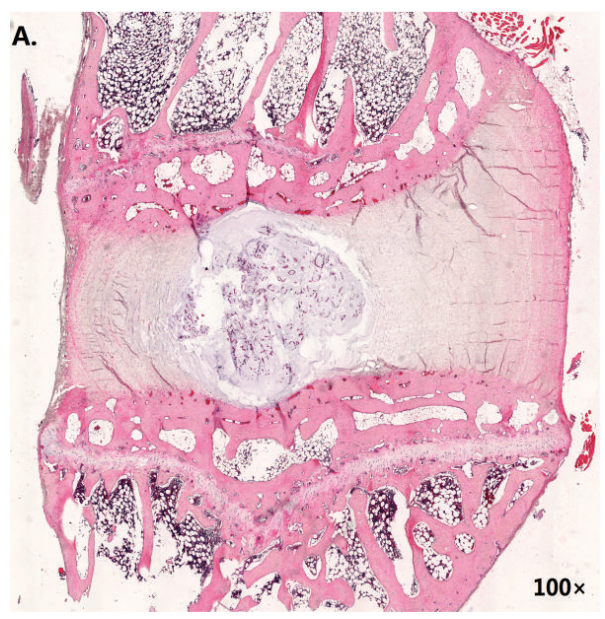

(a)

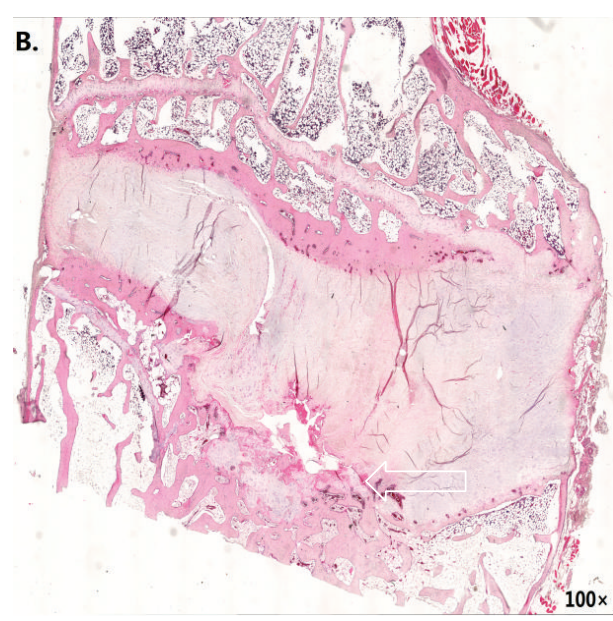

(b)

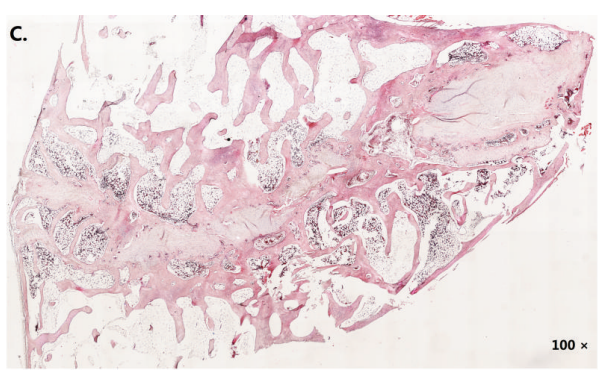

(c)

Figure 3: Eight weeks after the inoculation, P. acnes-inoculated intervertebral discs showed significant disc degeneration and endplates rupture but without severe discitis. (a) The intervertebral discs harvested from the TSB-inoculated internal control segment (L4 L5) had distinct nucleus pulposus and normal arranged annulus fibrosus. (b) The segment of wild-type strain of $P$. acnes-inoculated intervertebral discs (L6 L7) was demonstrated as disappearance of nucleus pulposus, endplates fracture (white arrow), disorganized annulus fibrosus, and partly cartilage proliferation. (c) By contrast, the intervertebral space significantly narrowed in S. aureus-infected intervertebral discs in coincidence with total cartilage tissue replacement and disappearance of nucleus pulposus and annulus fibrosus.

severe inflammatory signal changes were found at the $S$. aureus-inoculated segment (Figures $2(\mathrm{~g})$ and $2(\mathrm{~h})$ ).

3.2. Lower Death Rate in P. acnes-Inoculated Animals. All of the five $P$. acnes-inoculated animals remained alive until the end of follow-up. However, of the three S. aureus-inoculated rabbits, one died at the fourth week and another died at the fifth week (Table 1). Only one rabbit was still alive at the end of follow-up. Thus, the inoculation of $S$. aureus seemed more fatal than that caused by $P$. acnes $(2 / 3$ versus $0 / 5$, Table 1$)$.

3.3. Disc Degeneration and Endplates Rupture at P. acnesInoculated Segment. In histological examination, TSBinoculated intervertebral discs showed normal anatomical structure, in which nucleus pulposus was enclosed with normal annulus fibrosus (Figure 3(a)). However, P. acnesinoculated segment was depicted as endplates rupture and moderate disc degeneration with the disappearance of nucleus pulposus and disorganizing of annulus fibrosus (Figure 3(b)). Comparatively, intervertebral discs infected with $S$. aureus showed significantly narrowed intervertebral space and annulus fibrosus was completely replaced by cartilage, indicating severe pathological change by discitis (Figure 3(c)). Thus, it was reasonable to claim that $P$. acnes inside intervertebral discs would result in disc degeneration and endplates rupture rather than the severe discitis.

\section{Discussion}

The wild-type strain of $P$. acnes isolated from the patient with disc degeneration and Modic change resulted in the same signal changes when inoculated into the intervertebral discs of rabbits. Associated with histological examination, the signal changes were more reasonable to be considered as Modic change and disc degeneration. Furthermore, compared to the higher death rate and severe pathological change of discitis caused by $S$. aureus, the inoculation of $P$. acnes inside intervertebral discs did not result in severe discitis. Therefore, it was strongly indicated that $P$. acnes was one of the pathogens for Modic change and disc degeneration.

Modic change is the abnormal signals at the endplates and subchondral bone on MRI, which is always secondary to the disc degeneration [1]. However, it may be similar to discitis on MRI, especially the Modic-I change, and it is not easy 
to distinguish them in certain cases. Conventionally, typical pyogenic discitis will show hyperintense signals involving the intervertebral discs and vertebrae on T2WI and defect or erosion at endplates and vertebral body with/without the abscess surrounding the intervertebral discs [19]. Nevertheless, for most atypical discitis and discitis in early stage, hyperintense signals may only involve the endplates and subchondral bone but without any abnormality in disc and surrounding soft tissue [20]. Even in some reported S. aureus-infected rabbit models, no hyperintense signal changes were observed at intervertebral discs and surrounding soft tissue, which was also similar to this study's result [17]. Here, in this study, the outline of the endplates is more intact in P. acnes-inoculated segment and the inflammatory oedema seemed more severe in S. aureus-inoculated segment so that we are inclined to the Modic changes rather than discitis in P. acnes-inoculated segment.

Based on Koch's Postulates, we further believed that $P$. acnes was one of the pathogens causing Modic changes and disc degeneration. As one of the most important criteria to judge the relationship between a specific microbe organism and a specific disease, "finding abundant microorganism in organisms suffering from the disease" and "isolating and culturing this organism in pure culture medium" were the first and second criteria, and "cultured microorganism could cause disease when introduced into a healthy organism" and "re-isolated from the inoculated, diseased experimental host" were the third and fourth steps [21]. Previous study has demonstrated the existence of $P$. acnes in the nonpyogenic degenerated intervertebral discs $[6,8]$, and our previous study also proved a prevalence of $23.9 \%$ in degenerated discs [16]. Here, the MRI and histological evidence (Figures 2 and 3 , resp.) further suggested that inoculated $P$. acnes inside animal's intervertebral discs would cause Modic change and disc degeneration. All of these data satisfied the first, second, and third criteria of Koch's Postulates and thus we could extrapolate that $P$. acnes has a strong connection with Modic change and disc degeneration.

Being different from $S$. aureus, which was well known as the most frequent pathogen in spondylodiscitis [22], $P$. acnes rarely caused pyogenic discitis. In a thorough review, only twenty-nine cases of $P$. acnes-induced spondylodiscitis were reported within the last 15 years [23]. On the contrary, the prevalence of $P$. acnes in nonpyogenic intervertebral discs was as high as $44 \%$ [6] and a similar prevalence was independently reported by other different research groups $[7,8]$. One of the possible reasons may be the fact that $P$. acnes had different species and some of them are more virulent, but others may be less virulent [11], thus probably leading to different pathological change and dispersion of MRI image. Generally speaking, we favored that when the intervertebral discs are infected by $P$. acnes they would have more chance to result in the latent low-grade chronic inflammation [6], which is then represented as Modic changes and disc degeneration rather than the pyogenic discitis.

Recently, an illuminating animal paper tried to reveal the routine of how $P$. acnes gets access into the intervertebral discs [18]. Although the results did not support the hypothesis proposed by Albert et al. that $P$. acnes may intrude the intervertebral discs via circulation system [9], it proved that intervertebral discs are suitable for the growth and reproduction of $P$. acnes, because the reisolation percentage was as high as $61 \%$ after the inoculation of P. acnes directly into the intervertebral discs. More importantly, disc degeneration was also observed on the MRI after $P$. acnes inoculation, which was consistent with our results [18].

Unfortunately, it is still unclear how $P$. acnes induces the Modic changes. One viewpoint was that the propionic acid, the metabolite secreted by $P$. acnes, resulted in the dissolution of fatty bone marrow and bone, thus represented as Modic changes [10]. Another hypothesis was the local inflammatory activated by $P$. acnes [9]. P. acnes has been proved to have a strong ability to stimulate monocytes to produce proinflammatory factors such as TNF- $\alpha$ and IL-1 $\beta$ [24], so that these proinflammatory factors may be involved in the damage and oedema of endplates and subchondral bone marrow, which is displayed as Modic-I change on MRI [25]. Also, increased proinflammatory factors would lead to the disc degeneration [26].

During the isolation of $P$. acnes from human being, we followed previous method: muscle and ligaments were extracted as the contamination marker to avoid false-positive culture result $[12,16]$. In addition, the reason that we used adjacent intervertebral disc for TSB inoculation is that it will keep the bacterium-inoculated and TSB-inoculated segments having the same surgical condition. Moreover, the purpose that sterilized TSB instead of saline was used as the solvent to prepare $P$. acnes suspension was to maintain bacterial viability. Although macromolecular substances in the TSB may have unpredictable effect, the TSB alone inoculation segment did not show any abnormal changes, suggesting that TSB used as the solvent was a practicable option. Finally, according to previous study, the penetration with 28-gauge needle had minor or no effect on disc degeneration in rabbits [27].

However, some limitations still exist in this experiment. First, other low-virulence anaerobic floras, like coagulasenegative staphylococci, were not tested in this experiment. In addition, observation duration was relatively short in this study. Long-term effect of $P$. acnes on intervertebral discs and endplates requires further study.

\section{Conclusion}

Based on Koch's Postulates, P. acnes has strong correlation with disc degeneration and Modic changes when inoculated into intervertebral discs, which is definitely different from discitis caused by S. aureus in histological examination and death rate. In clinical practice, one should be more aware of the presence of $P$. acnes in patients with Modic changes and disc degeneration.

\section{Conflict of Interests}

The authors declare that there is no conflict of interests regarding the publication of this paper. 


\section{Authors' Contribution}

Zhe Chen and Yuehuan Zheng contributed equally to this work.

\section{Acknowledgments}

This work was supported by grants from the Science and Technology Commission of Shanghai Municipality, Shanghai, China (no. 13430722100 and no. 15DZ1942604) and grants from the Shanghai Bureau of Health, Shanghai, China (no. XBR2011024).

\section{References}

[1] M. T. Modic and J. S. Ross, "Lumbar degenerative disk disease," Radiology, vol. 245, no. 1, pp. 43-61, 2007.

[2] Y.-H. Zhang, C.-Q. Zhao, L.-S. Jiang, X.-D. Chen, and L.-Y. Dai, "Modic changes: a systematic review of the literature," European Spine Journal, vol. 17, no. 10, pp. 1289-1299, 2008.

[3] M. T. Modic, P. M. Steinberg, J. S. Ross, T. J. Masaryk, and J. R. Carter, "Degenerative disk disease: assessment of changes in vertebral body marrow with MR imaging," Radiology, vol. 166, no. 1, part 1, pp. 193-199, 1988.

[4] M. Kuisma, J. Karppinen, J. Niinimäki et al., "Modic changes in endplates of lumbar vertebral bodies: prevalence and association with low back and sciatic pain among middle-aged male workers," Spine, vol. 32, no. 10, pp. 1116-1122, 2007.

[5] J. H. Määttä, S. Wadge, A. MacGregor, J. Karppinen, and F. M. Williams, "ISSLS prize winner: vertebral endplate (Modic) change is an independent risk factor for episodes of severe and disabling low back pain," Spine, vol. 40, no. 15, pp. 1187-1193, 2015.

[6] A. Stirling, T. Worthington, M. Rafiq, P. A. Lambert, and T. S. J. Elliott, "Association between sciatica and Propionibacterium acnes," The Lancet, vol. 357, no. 9273, pp. 2024-2025, 2001.

[7] J. Arndt, Y. P. Charles, C. Koebel, I. Bogorin, and J.-P. Steib, "Bacteriology of degenerated lumbar intervertebral disks," Journal of Spinal Disorders and Techniques, vol. 25, no. 7, pp. E211-E216, 2012.

[8] H. B. Albert, P. Lambert, J. Rollason et al., "Does nuclear tissue infected with bacteria following disc herniations lead to Modic changes in the adjacent vertebrae?" European Spine Journal, vol. 22, no. 4, pp. 690-696, 2013.

[9] H. B. Albert, P. Kjaer, T. S. Jensen, J. S. Sorensen, T. Bendix, and C. Manniche, "Modic changes, possible causes and relation to low back pain," Medical Hypotheses, vol. 70, no. 2, pp. 361-368, 2008.

[10] H. B. Albert, J. S. Sorensen, B. S. Christensen, and C. Manniche, "Antibiotic treatment in patients with chronic low back pain and vertebral bone edema (Modic type 1 changes): a double-blind randomized clinical controlled trial of efficacy," European Spine Journal, vol. 22, no. 4, pp. 697-707, 2013.

[11] A. Perry and P. Lambert, "Propionibacterium acnes: infection beyond the skin," Expert Review of Anti-Infective Therapy, vol. 9, no. 12, pp. 1149-1156, 2011.

[12] A. Carricajo, C. Nuti, E. Aubert et al., "Propionibacterium acnes contamination in lumbar disc surgery," Journal of Hospital Infection, vol. 66, no. 3, pp. 275-277, 2007.
[13] J. O’Dowd and A. Casey, "Antibiotics a cure for back pain, a false dawn or a new era?” European Spine Journal, vol. 22, no. 8, pp. 1694-1697, 2013.

[14] D. N. Fredricks and D. A. Relman, "Sequence-based identification of microbial pathogens: a reconsideration of Koch's postulates," Clinical Microbiology Reviews, vol. 9, no. 1, pp. 1833, 1996.

[15] R. Koch, "Uber bakteriologische Forschung," in Verhandlung des X Internationalen Medichinischen Congresses, Berlin, 1890, August Hirschwald, Berlin, Germany, 10th International Congress of Medicine, Berlin, Germany, 1891 (German).

[16] Z. Zhou, Z. Chen, Y. Zheng et al., "Relationship between annular tear and presence of Propionibacterium acnes in lumbar intervertebral disc," European Spine Journal, vol. 24, no. 11, pp. 2496-2502, 2015.

[17] G. Bierry, F. Jehl, G. Prévost et al., "Percutaneous inoculated rabbit model of intervertebral disc space infection: magnetic resonance imaging features with pathological correlation," Joint Bone Spine, vol. 75, no. 4, pp. 465-470, 2008.

[18] B. Li, Z. Dong, Y. Wu et al., "between lumbar disc degeneration and Propionibacterium acnes infection: clinical research and preliminary exploration of animal experiment," Spine, 2015.

[19] S. H. Hong, J.-Y. Choi, J. W. Lee, N. R. Kim, J.-A. Choi, and H. S. Kang, "MR imaging assessment of the spine: infection or an imitation?" Radiographics, vol. 29, no. 2, pp. 599-612, 2009.

[20] J. A. T. Dunbar, J. A. T. Sandoe, A. S. Rao, D. W. Crimmins, W. Baig, and J. J. Rankine, "The MRI appearances of early vertebral osteomyelitis and discitis," Clinical Radiology, vol. 65, no. 12, pp. 974-981, 2010.

[21] A. S. Evans, "Causation and disease: the Henle-Koch postulates revisited," Yale Journal of Biology and Medicine, vol. 49, no. 2, pp. 175-195, 1976.

[22] J. S. Butler, M. J. Shelly, M. Timlin, W. G. Powderly, and J. M. O'Byrne, "Nontuberculous pyogenic spinal infection in adults: a 12-year experience from a tertiary referral center," Spine, vol. 31, no. 23, pp. 2695-2700, 2006.

[23] I. Uçkay, A. Dinh, L. Vauthey et al., "Spondylodiscitis due to Propionibacterium acnes: report of twenty-nine cases and a review of the literature," Clinical Microbiology and Infection, vol. 16, no. 4, pp. 353-358, 2010.

[24] M. D. Farrar and E. Ingham, "Acne: inflammation," Clinics in Dermatology, vol. 22, no. 5, pp. 380-384, 2004.

[25] S. Ohtori, G. Inoue, T. Ito et al., "Tumor necrosis factorimmunoreactive cells and PGP 9.5-immunoreactive nerve fibers in vertebral endplates of patients with discogenic low back pain and modic type 1 or type 2 changes on MRI," Spine, vol. 31, no. 9, pp. 1026-1031, 2006.

[26] M. V. Risbud and I. M. Shapiro, "Role of cytokines in intervertebral disc degeneration: pain and disc content," Nature Reviews Rheumatology, vol. 10, no. 1, pp. 44-56, 2014.

[27] D. M. Elliott, C. S. Yerramalli, J. C. Beckstein, J. I. Boxberger, W. Johannessen, and E. J. Vresilovic, "The effect of relative needle diameter in puncture and sham injection animal models of degeneration," Spine, vol. 33, no. 6, pp. 588-596, 2008. 


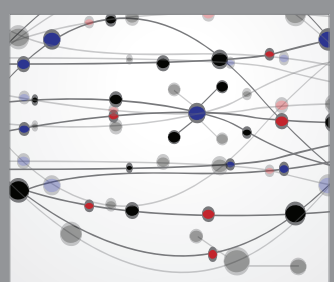

The Scientific World Journal
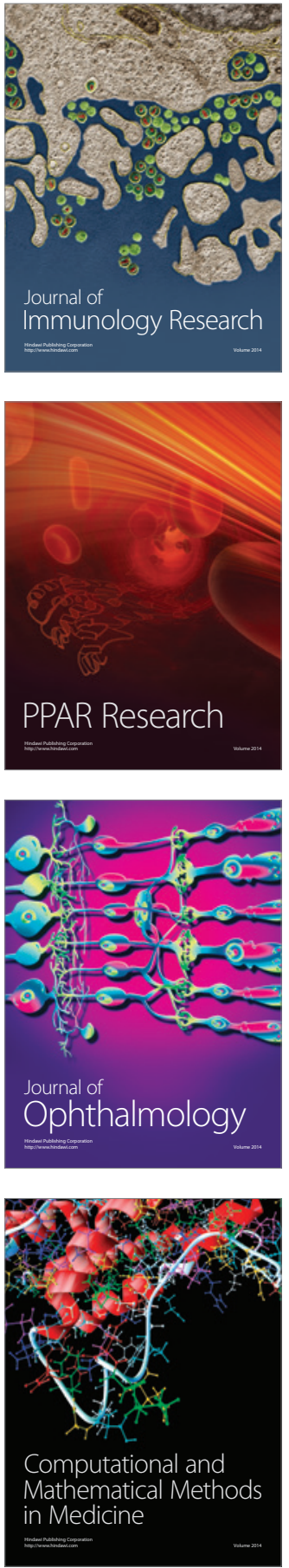

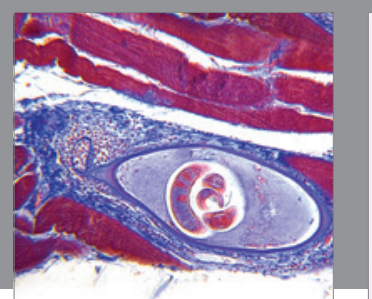

Gastroenterology Research and Practice

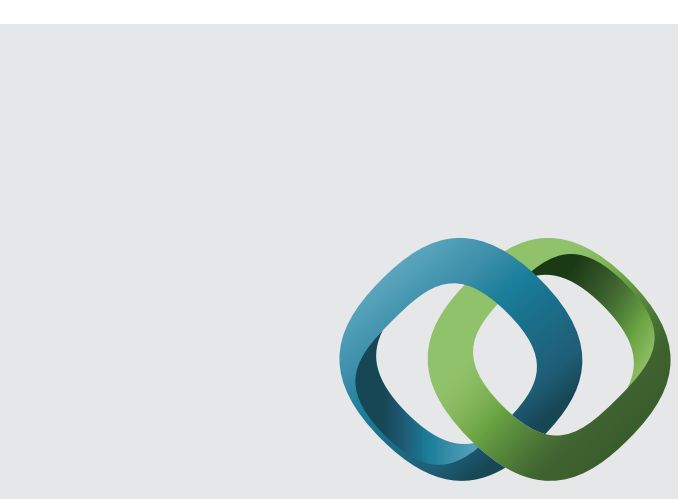

\section{Hindawi}

Submit your manuscripts at

http://www.hindawi.com
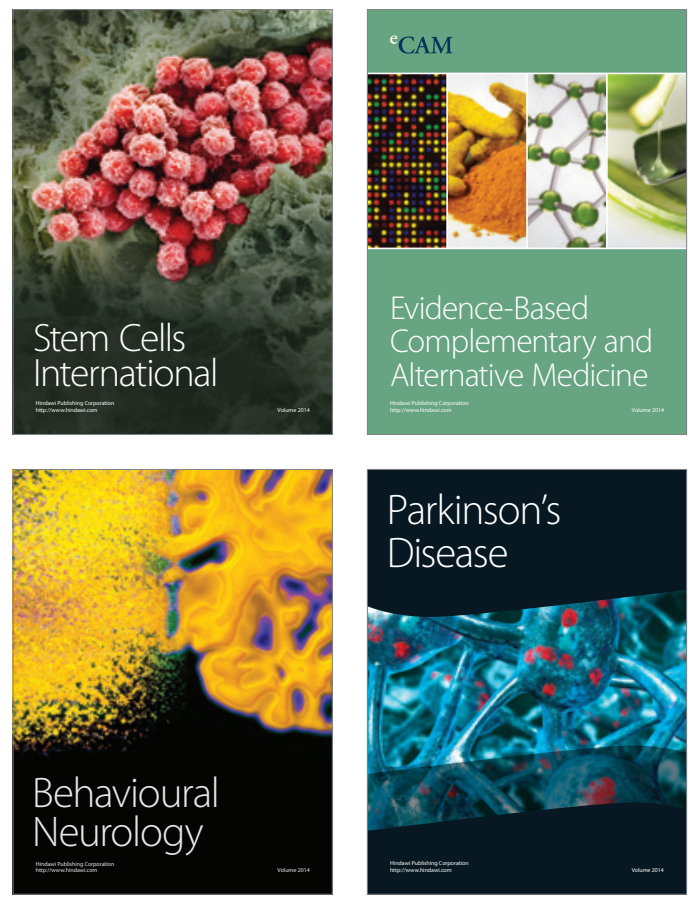
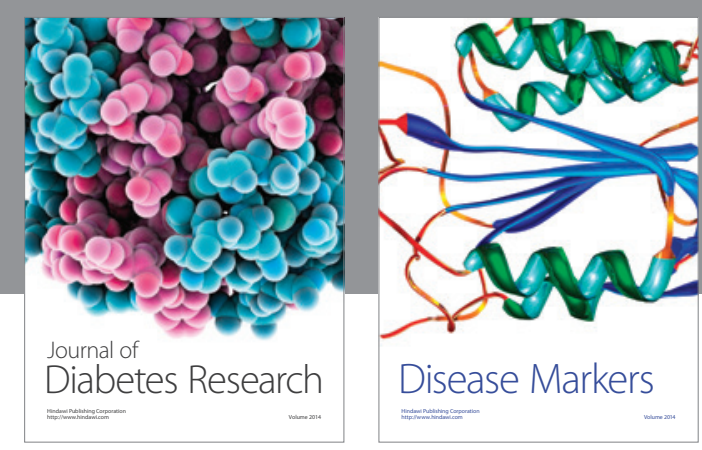

Disease Markers
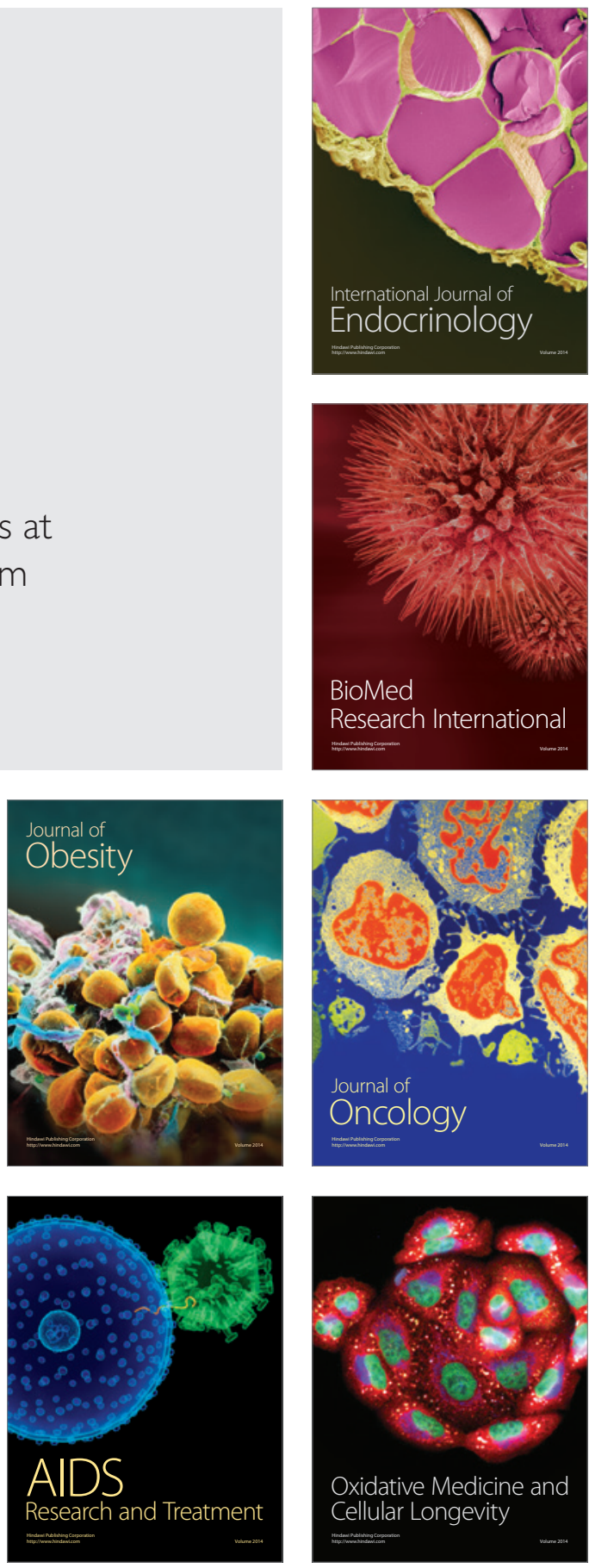